\title{
Dans l'intimité de la Vierge. Dévotions au féminin et au masculin en Grèce contemporaine
}

\section{Katerina Seraïdari}

\section{(2) OpenEdition}

\section{Journals}

Édition électronique

URL : https://journals.openedition.org/clio/59

DOI : $10.4000 /$ clio. 59

ISSN : 1777-5299

Éditeur

Belin

Édition imprimée

Date de publication : 1 avril 2002

Pagination : 55-68

ISBN : 2-85816-620-X

ISSN : 1252-7017

\section{Référence électronique}

Katerina Seraïdari, « Dans l'intimité de la Vierge. Dévotions au féminin et au masculin en Grèce contemporaine », Clio. Histoire, femmes et sociétés [En ligne], 15 | 2002, mis en ligne le 08 février 2005, consulté le 30 avril 2022. URL : http://journals.openedition.org/clio/59 ; DOI : https://doi.org/10.4000/ clio.59 


\section{Dans l'intimité de la Vierge. Dévotions au féminin et au masculin en Grèce contemporaine}

Katerina SERAIDARI

Construit à l'extrémité du village de Mandraki, sur un rocher surplombant la mer, le monastère de la Panayia Spiliani ${ }^{1}$ domine le chef-lieu de Nissyros. Cette petite île volcanique du Dodécanèse, qui compte à peine huit cent cinquante habitants, est placée sous sa haute protection. Son emplacement lui a d'ailleurs valu le titre d' "acropole de Nissyros" et de "Météores du Dodécanèse». En effet, il faut gravir cent trente marches pour y parvenir, puis descendre encore quelques escaliers afin de pénétrer dans l'église. Cet espace souterrain, qui rappelle les catacombes, abrite l'icône miraculeuse de la Vierge. Pour sa fête, le 15 août, près de cinq mille pèlerins viennent lui rendre hommage chaque année.

Si le nombre de visiteurs varie selon les estimations, les ex-voto qui sont accrochés au-dessous de l'icône sont la preuve concrète de la dévotion qu'elle suscite. Ces offrandes évoquent le plus souvent la vie familiale. Ainsi peut-on distinguer des bébés de cire qui, selon le pope du sanctuaire, sont offerts par des femmes stériles priant pour avoir une chance d'accéder à la maternité. Mais la Panayia Spiliani est surtout sollicitée par les mères pour protéger leurs enfants. Et c'est pour la remercier de sa bien-

1 Panayia signifie Vierge en grec ; Spiliani est le vocable de cette Vierge locale et signifie "de la grotte». 
veillance que les femmes interviennent de manière active dans la fête organisée en l'honneur de sa Dormition'.

Les femmes de l'île ne sont pas les seules à y être impliquées; d'autres viennent de la grande île voisine, Kos, et tiennent également une place importante. Bien que Nissyros attende ces festivités estivales pour s'animer, Kos constitue un pôle touristique considérable et est dotée d'un aéroport et d'une population de quinze mille habitants. Les pèlerines, qui laissent leurs maisons à Kos pour venir habiter dans les cellules du monastère de Spiliani pendant neuf jours, ne sont pourtant pas associées à cette image de confort matériel et de prospérité ; ce qui les caractérise est leur comportement pénitentiel, les trois cents génuflexions qu'elles doivent accomplir chaque jour devant l'icône mariale. Elles arrivent à Nissyros le 6 août (jour de la Transfiguration) et en repartent l'après-midi du 15 . On les appelle niameritisses. Leur nom, qui signifie «de la neuvaine», fait d'elles un groupe homogène bien que chacune soit liée à la Vierge par une promesse personnelle.

Tandis que les femmes s'approprient l'espace sacré par leur dévotion, les hommes choisissent comme lieu d'activité le centre du village et la préparation des grands festins qui y sont donnés les 14 et 15 août. Dans ce cadre, chaque sexe essaie non seulement de forger son image, mais aussi de souligner sa position centrale dans la fête et par rapport à la sainte patronne. L'analyse de cette répartition des rôles ${ }^{3}$ nous permettra de mieux comprendre la manière dont femmes et hommes construisent leur

2 Il s'agit de la mort de la Vierge, dont le déroulement rappelle celle du Christ. Selon la tradition orthodoxe, la Vierge aurait connu une mort humaine qui lui aurait été prédite par un ange. Lors de son agonie dans le jardin de Gethsémani, elle pleura et demanda à mourir près du sépulcre de son Fils. Après son trépas, son corps fut enseveli à Gethsémani et sa sépulture close par une pierre. Trois jours plus tard, en présence du Christ, des anges emportèrent alors son corps au Paradis.

3 Il est évident que tous les hommes ou toutes les femmes ne participent pas de la même manière aux festivités. Ici, j'examine la norme et non pas les cas divergents : bien que cette approche amène à reproduire les stéréotypes que mes informateurs véhiculent dans leurs discours, elle révèle également leur manière de percevoir la fête. J'ai effectué mes enquêtes à Nissyros en août 1997 et en avril 1998. Pour une plus ample information sur cette fête, voir Seraïdari 2000. 
relation avec la Vierge et revendiquent leur part $d u$ sacré. Cependant, ce n'est pas seulement le déroulement des festivités qui laisse apparaître un tel partage, mais aussi les récits qui fondent la réputation de la Panayia Spiliani. Du rituel aux légendes, ce parcours révèle, d'une part, les liens de complicité qui unissent les femmes et la mère du Christ ; et d'autre part, le rapport qu'entretient la patronne de Nissyros avec un domaine principalement masculin, la consommation rituelle de vin et de nourriture.

\section{Le monastère, un espace féminin}

De grande taille, l'icône de Spiliani est installée entre les colonnes soutenant l'architrave de l'iconostase. Dans les églises orthodoxes, l'iconostase vise à isoler l'autel où le pope célèbre la communion. Généralement au nombre de quatre, les icônes qui y sont placées représentent le Christ, la Vierge, saint Jean-Baptiste, ainsi que le saint patron de l'église. La tradition veut que l'icône de la Vierge soit placée à gauche et l'icône du Christ à droite (du point de vue des fidèles). Cette disposition réglemente aussi la place des femmes et des hommes dans l'église : les femmes restent à gauche ${ }^{4}$, devant l'icône de la Vierge, et les hommes à droite, devant l'icône du Christ. Pourtant, dans le sanctuaire de Nissyros la position des icônes est inversée : l'icône de la Panayia Spiliani se trouve à droite et celle du Christ à gauche. À ma question de savoir le pourquoi de cette organisation inhabituelle de l'espace, une jeune femme de l'île m'a répondu que "c'est peut-être pour honorer encore plus la Vierge». Comme les femmes sont obligées de rester en face de son icône, elles occupent dans cette église la place qui appartient traditionnellement aux hommes.

Cette spécificité semble encore plus étonnante, si l'on prend en compte le fait que le monastère est depuis longtemps habité par des moines 5 .

4 Selon Jill Dubisch (1983: 128), étant donné la connotation négative du côté gauche, cette distribution de l'espace sacré reflète le statut inférieur assigné aux femmes. Laurie Hart (1992 : 113) constate la même tendance, mais elle suggère également qu'en mettant la Vierge près de la main droite du Christ, on vise à souligner la position ambiguë de celle-ci et, par conséquent, de toutes les femmes.

5 Selon un érudit local, des documents font référence à l'higoumène de Spiliani depuis 1600 (Kentris, 1991 : 13). L'higoumène est le supérieur d'une communauté monas- 
Aujourd'hui, il ne reste sur place qu'un archimandrite (titre monastique) qui est en même temps le pope de Mandraki. Cet endroit, où plus aucune communauté monastique ne réside à demeure, reprend donc vie grâce aux niameritisses. Elles se partagent les cellules, font le ménage et préparent leur nourriture quotidienne ; elles jeûnent aussi toutes ensemble afin de recevoir la communion la veille de la Dormition. Ces femmes comblent, en quelque sorte, l'absence actuelle des religieux.

Si le rapprochement entre les niameritisses et la communauté monastique du passé ne peut se faire que pendant la fête, il existe une autre figure féminine à qui les habitants ont attribué le titre de "nonne». Il s'agit d'une femme qui est restée légendaire : bien qu'elle n'eût pas un statut religieux officiel, cette désignation indique la reconnaissance populaire de sa position par rapport à la Vierge. En effet, on raconte que la «nonne» regardait l'icône et savait instantanément si la Vierge était là ou si elle était partie pour secourir un dévot. Car elle parlait avec la Panayia Spiliani. Cette dernière lui avait dit que si son visage était plus obscur («skouro»), cela signifiait qu'elle était présente ; si, au contraire, son visage était clair, elle était absente, elle ne pouvait donc ni entendre ni aider ceux qui s'adressaient à elle à ce moment-là. Selon mes informateurs, cette explication fut donnée par la «nonne» à l'occasion de la mort d'un enfant, dont la mère était venue en vain demander la guérison à la Panayia Spiliani alors «absente».

Sakellaridis (1982 : 305) donne quelques éléments biographiques concernant cette femme : elle aurait été une jeune niameritissa, qui, attirée par la vie dans le monastère, aurait décidé d'y rester pendant plusieurs années comme "fille de la Vierge" ${ }^{6}$. Lauteur cite également une histoire qui montre comment elle mettait son don au service de la communauté locale. En 1873, une épidémie de rougeole ravageait l'île ; un jour, la

tique masculine, équivalent d'un abbé.

6 La nestinarka est, dans l'est de la Thrace bulgare, une femme âgée qui entre dans le feu et danse pour honorer saint Constantin ; selon une croyance populaire, elle est considérée comme la "sœur du saint" (Georgieva, 2001 : 256). Dans les deux cas, ces femmes, qui acquièrent un statut important dans la communauté locale grâce à leur dévotion, se voient dotées des liens généalogiques avec leur saint patron. 
jeune femme sortit dans le village pour annoncer que la Vierge avait quitté l'île pendant ce temps-là et que, maintenant qu'elle était de retour, les malades guériraient et l'épidémie s'arrêterait, ce qui ne manqua pas de se réaliser.

Kentris (1991 : 28) rapporte la même histoire, mais il laisse entendre que la Vierge revint sur Nissyros grâce aux prières incessantes de la "nonne». Il fait d'ailleurs référence à un autre miracle : la punition divine d'un pirate qui, en 1820 , voulut voler les ex-voto d'or et d'argent de l'icône ; là aussi, ce fut la «nonne» qui lui indiqua ce qu'il fallait faire pour sauver sa vie (Kentris 1991 : 24). Si celle-ci était déjà dans le monastère en 1820 , comment est-il possible que Sakellaridis la présente comme une jeune femme en 1873 ? D'autant plus qu'une niameritissa m'a raconté le miracle suivant : lors de la seconde guerre mondiale, la Vierge fit fuir les soldats allemands qui voulaient s'installer dans le monastère et qui venaient de maltraiter la «nonne». La juxtaposition de ces récits révèle la tendance à mettre systématiquement en relation toute intervention miraculeuse avec une figure féminine plus ou moins mythique. Le fait d'interposer entre les dévots (ou ceux qui commettent un sacrilège) et les représentants officiels de l'Église, une femme et plus particulièrement une ex-niameritissa, montre les liens privilégiés qui sont noués entre la Vierge et ces pèlerines; le destin singulier de la "nonne" ne fait que confirmer leur statut particulier.

En effet, ce sont elles qui profitent le plus souvent d'un miracle ou d'une apparition. Cela n'est pas étonnant, dans la mesure où elles habitent pendant neuf jours dans la «maison de la Vierge», le lieu par excellence où celle-ci manifeste son caractère miraculeux. Ainsi dit-on que lorsque les niameritisses les plus âgées restent dans l'église tard le soir, elles entendent parfois le bruit des sabots ("tsokara") de la Panayia Spiliani qui fait le tour de son monastère. En revanche, l'histoire qui circule sur le précédent pope appartient à un tout autre registre : il hésitait à aller tout seul dans le sanctuaire pour allumer la veilleuse, de peur d'y rencontrer la Vierge se promenant. Il se faisait toujours accompagner par son épouse («tin papadia tou») ! Souligner la crainte que la Vierge inspire même à un clerc signifie, tout d'abord, insister sur sa présence active qui «hante» le 
monastère ; néanmoins, ce récit montre aussi que le pope n'avait pas la même familiarité que les niameritisses avec la sainte patronne.

Le pope actuel, lui non plus, n'a pas une relation "personnelle» avec la Vierge. Selon une niameritissa, il aurait entendu une fois des cris d'enfant venant du sanctuaire ; il était tard le soir du jour de l'Annonciation, la messe était finie depuis longtemps et il s'apprêtait à dormir dans sa cellule au monastère. Il descendit dans l'église où il constata qu'il n'y avait personne et que les pleurs résonnaient encore. "Une mère doit avoir son bébé malade et l'a invoquée auprès d'elle», fut l'explication que le pope donna de ce phénomène. La niameritissa conclut son histoire en disant : "Un jour, cette femme viendra pour remercier la Vierge et on saura alors ce qui s'est réellement passé». Dans ce cas, le pope n'est qu'un témoin à qui une partie seulement de l'action divine est révélée. Malgré sa place dans l'institution ecclésiastique, il n'est pas capable, comme la «nonne» l'était, de jouer le rôle de médiateur entre la patronne locale et celles qui la sollicitent. Certes, il sert la messe en son honneur et observe les manifestations surnaturelles sur lesquelles sa réputation est fondée ; mais il ne peut parler avec certitude de sa volonté et du secours qu'elle apporte aux fidèles.

À la complicité qui unit les femmes ${ }^{7}$ et la Vierge s'oppose la distance que les hommes gardent par rapport à ce qui se passe dans l'enceinte du monastère. Ceux-ci assistent éventuellement à l'office, mais ils ne font jamais de génuflexions ; comme une niameritissa me l'a dit, "les hommes sont très fiers, ils n'aiment pas s'incliner devant l'icône". Leur contact avec l'icône étant limité et impersonnel, seule la préparation de la nourriture festive leur permet de se placer du côté du sacré. Ils expriment, en effet, leur dévotion à la Panayia Spiliani par cet autre biais, l'organisation des festins qui clôturent les célébrations.

7 Les femmes de l'île effectuent elles aussi des génuflexions pendant cette période. Ce qui les différencie pourtant des niameritisses est le fait que ces dernières séjournent dans le monastère et, par conséquent, adoptent un rythme de vie monacal. 


\section{Quand les hommes font la fête}

Tandis que tout au long de l'année, ce sont les femmes qui font la cuisine dans l'espace domestique, cette tâche est confiée aux hommes de Mandraki lors des festivités collectives. La justification de cette répartition est que, quand des grandes quantités sont nécessaires, seuls les hommes ont la force physique requise pour soulever et manipuler les lourds chaudrons. Dans ce contexte, prendre en charge le côté matériel de la fête devient un acte d'affirmation de virilité et d'engagement dans la vie communautaire. Cette inversion des rôles marque ainsi la différence entre les moments ordinaires que l'on passe dans le cercle familial, et un temps festif et collectif. En effet, l'inversion des positions entre les deux sexes concernant le domaine culinaire conditionne le passage de la cellule familiale à la communauté toute entière.

Les femmes accomplissent leurs dernières génuflexions le 13 août. A partir de ce moment, la fête se déplace du monastère à la "salle à manger de la Vierge" ("Trapeza tis Panayias»), qui se trouve tout près de la place centrale de Mandraki. Il s'agit d'une maison traditionnelle qui servait auparavant d'école primaire et qui fut aménagée il y a quarante ans, grâce à la donation d'un émigré, de manière à ce que les festins puissent $y$ avoir lieu. L'icône de la Vierge participe à ce mouvement. Nous avons vu que dans un premier temps, on monte au monastère afin de lui rendre hommage ; après la messe matinale du 15 août, c'est l'icône qui est amenée en procession au centre de la communauté. On la fait même entrer dans la cuisine de la "salle à manger de la Vierge" pour qu'elle bénisse la nourriture, afin que le festin puisse commencer. Après avoir mangé, les convives font la queue pour saluer l'icône qui est posée sur une table, à la sortie de la cour. C'est donc elle qui ouvre et marque la fin du banquet.

Sa présence au milieu des convives non seulement confirme le caractère religieux du rassemblement, mais permet aussi aux hommes de donner un sens pieux à leur contribution. En faisant la cuisine et en servant la foule des pèlerins, ceux-ci honorent la sainte patronne à leur façon. Néanmoins, la réalisation du festin ne dépend pas exclusivement d'eux; la Vierge peut aussi bien donner sa bénédiction que provoquer une catas- 
trophe. L'histoire, qui explique pourquoi les habitants revinrent sur la décision de ne plus sortir l'icône originale, va dans ce sens. On raconte que l'année où une copie de l'icône prit sa place, un accident eut lieu : dès que la copie arriva dans la cuisine, un chaudron plein de pommes de terre se renversa soudainement, provoquant un début d'incendie.

Ce récit souligne la réaction de la Panayia Spiliani contre l'introduction d'une nouveauté dans la fête. Les hommes se sont assignés un devoir semblable : garder les coutumes intactes et assurer leur perpétuation. Ainsi, ils ont eu à s'opposer au Service Archéologique d'Athènes qui voulait interdire l'utilisation de cette maison traditionnelle pour les banquets communautaires. Ils critiquent également vivement l'attitude de la police locale qui intervient chaque année pour faire arrêter la fête à minuit. À travers leur action et leurs discussions publiques, les hommes se présentent comme les défenseurs de l'héritage culturel local. En effet, si les liens entre la Vierge et ses dévotes sont construits dans la discrétion, au travers de voeux évoquant des soucis familiaux, c'est la détermination à préserver la tradition ancestrale contre toute menace extérieure qui rapproche la population masculine de la sainte patronne.

Une fresque peinte sur un mur de la cour de la «salle à manger de la Vierge» montre saint Georges tuant le dragon. Sur sa lance est écrit le mot «tradition» («paradossi») et, sur le corps du dragon, «oubli» et «aliénation» ( (lismossini, allotriossi»). Cette représentation anticipe la victoire des coutumes locales sur une modernité changeante. En même temps, la référence à ce saint guerrier permet aux hommes de s'identifier à un modèle qui est, en même temps, religieux et viril.

Sur le portail de ce même bâtiment figure aussi une inscription qui met en relation les festins avec les agapes des premiers chrétiens. Comme ce sont les hommes qui s'occupent des banquets, on peut penser que ce rapprochement constitue pour eux une autre manière de se rattacher, à la fois, à un symbole religieux et au passé. En évoquant une des premières pratiques chrétiennes, non seulement on fait allusion à la solidarité et à l'esprit égalitaire de la fête, mais on magnifie aussi la contribution masculine : c'est grâce à leurs efforts que la communauté locale peut se vanter d'avoir atteint l'idéal chrétien, faire revivre chaque été le temps des ori- 
gines du christianisme. Par leur biais, la tradition locale et l'histoire du christianisme deviennent ainsi indissociables.

La "salle à manger de la Vierge" peut donc être considérée comme un prolongement du monastère : investie de sens multiples, elle représente à la fois un champ de bataille (entre le Bien et le Mal qu'incarne le dragon vaincu par saint Georges, entre le «local» et le "global»), mais aussi un lieu de convivialité et de partage. En outre, les festins sont principalement financés par le monastère de Spiliani. Le premier est donné le soir du 14 août ; il consiste en un plat maigre, constitué de pois chiches bouillis dans de la sauce tomate et accompagné d'olives, de tomates et d'oignons. Le deuxième, le midi du 15 août, est le plus important : à chacun est servi un plat de viande cuite dans de la sauce tomate, accompagnée de frites et de riz. La présence de l'icône dans la cour, lors du repas carné, renforce l'impression que c'est elle qui régale, et que ce sont les hommes qui sont à son service. D'autant plus que les femmes participent à la préparation de la nourriture maigre, alors que ce sont eux qui s'occupent exclusivement de la viande et de sa cuisson.

En sacralisant, d'une part, les coutumes de l'île ${ }^{8}$ et d'autre part, la commensalité, les hommes parviennent à investir leur rôle d'une certaine spiritualité. Parfois, ils tiennent même des discours qui laissent entendre que leur participation à la fête est un sacrifice, un «don» d'énergie et de temps libre offert à la communauté. Ainsi, en parlant avec un émigré résidant à New York qui, une fois les festivités terminées, était prêt à entreprendre le long voyage de retour, un habitant n'a pas manqué de souligner la gratuité de leur contribution: "Tu as passé des heures et des heures à cuisiner, puis à servir tout ce monde et maintenant tu pars. Qui t'a remercié ? Qu'est-ce que tu as gagné en le faisant ?».

8 La "salle à manger de la Vierge" est pourtant équipée avec les derniers modèles de la technologie américaine. De même, les hommes qui s'occupent des festins sont, dans leur majorité, des émigrés qui viennent des États-Unis pour passer l'été sur leur île natale. Par exemple, un émigré a ramené un système mécanique pour tirer le chaudron du feu sans effort : tandis que les années précédentes, l'aide de plusieurs personnes était nécessaire pour cette tâche, tout se fait maintenant de manière automatique. 
Si les niameritisses deviennent pendant neuf jours les hôtesses qui accueillent les pèlerins dans le monastère, en revanche, les hommes de Mandraki sont responsables de leur réception lors des festins. Leur rôle dans la fête peut être mis en relation avec une légende décrivant la manière dont l'higoumène du monastère recevait jadis les visiteurs. Lors du repas, ceux-ci un peu gris ("pano sto kefi tous»)", jetaient leurs verres pleins de vin par la fenêtre, en faisant voeu d'offrir un verre d'argent de même taille à la Panayia Spiliani s'ils ne se cassaient pas après une chute de trente-cinq mètres. On raconte que les coupes restaient intactes après l'épreuve, et que même leur contenu n'était pas renversé. Grâce à cette série de miracles, l'icône possède aujourd'hui une collection de veilleuses d'or et d'argent en forme de verre. Ce récit associe donc le pouvoir miraculeux de l'icône à l'hospitalité du monastère et à la consommation du vin : nous sommes bien dans un domaine masculin, où les invités défient presque la Vierge et où les femmes ne sont plus médiatrices.

L'image d'abondance et de commensalité qui définit la contribution masculine dans la fête contraste avec le comportement pénitentiel des niameritisses. Lors de leur séjour au monastère, celles-ci sont soumises à un mode de vie austère qui inclut le jeûne, les génuflexions et la séparation de leur famille - surtout de ses membres masculins comme nous le verrons.

\section{Le temps des femmes : pénitences et transmission entre générations}

Comme une jeune femme de Mandraki me l'a dit, la niameritissa est une femme âgée, habillée en noir, avec un foulard lui couvrant la tête. Lors d'une autre discussion avec la même informatrice, j'ai appris que les niameritisses offrent souvent à l'icône des tissus destinés à être placés sur sa "tête" : cet "habillage" de l'icône renverrait à l'obligation que la population féminine avait auparavant de se couvrir la tête. D'ailleurs, selon le témoignage des femmes qui l'ont vue dans leur rêve, la Panayia Spiliani

9 Selon Evthymios Papataxiarchis (1994), le mot grec kefi, qui signifie la «bonne humeur", est lié à une forme de sociabilité masculine qui est mise en œuvre dans les cafés : boire en compagnie, chanter et danser. 
elle-même porte un couvre-chef. Dans ce cas, une certaine image féminine définit à la fois la Vierge locale, son icône et les niameritisses - ce qui renforce leur identification et fait de l'une le miroir de l'autre. En désignant la Vierge comme une personne ayant la tête couverte et en offrant des tels cadeaux à l'icône, les femmes la façonnent selon leur propre image ; en même temps, elles adoptent une apparence similaire à celle de leur sainte patronne ${ }^{10}$.

Les vêtements des niameritisses soulignent leur piété et le refus temporaire de leur féminité. Pourtant, de jeunes pèlerines habillées en jeans viennent, chaque année plus nombreuses, de Kos, pour séjourner pendant les neuf jours au monastère. Les habitants de Nissyros les regardent avec méfiance, soutenant que leur intention est tout simplement de prendre des vacances. En fait, ce n'est pas seulement le choix vestimentaire de ces femmes qui les éloignent du modèle classique de la niameritissa, mais aussi leur âge ${ }^{11}$. Car la niameritissa est le plus souvent une grand-mère qui amène avec elle sa petite-fille ${ }^{12}$ : les grands-mères actuelles y sont venues pour la première fois, elles aussi, comme petites-filles accompagnées par leurs propres grands-mères.

Tandis que les hommes défendent le droit de continuer de faire la fête de la même manière que leurs aïeux l'ont faite, les niameritisses sont en revanche associées à un autre héritage : l'expression d'une piété qu'elles

10 Pour une analyse de la manière dont les femmes "font" la Vierge et se "font» ellesmêmes à travers la coutume de vêtir les statues mariales dans le monde catholique, voir Marlène Albert-Llorca 1995.

11 Il faut dire aussi que c'est leur façon de s'habiller qui «rend âgées» les niameritisses (de la même manière que les jeans sont considérés comme des vêtements de jeune). D'ailleurs, le terme grec qui signifie "nonne" ("kaloyria») se traduit littéralement par «bonne vieille».

12 Lors de ses enquêtes sur le pèlerinage marial de l'île cycladique de Tinos, Jill Dubisch (1991 : 37) a constaté que plusieurs grands-mères y venaient accompagnées par leurs petits-enfants. Dans cet article, J. Dubisch examine le rôle actif des femmes dans la structuration de la parenté et des réseaux familiaux, mais aussi dans le champ religieux. Elle propose même de considérer la "religion populaire» en Grèce comme une création féminine qui s'oppose à la religion officielle - domaine où les hommes ont une place dominante. 
partagent avec celles qui les ont précédées. Dans le monastère, on montre une dalle de marbre creusée à plusieurs endroits, qui se trouve à gauche de l'icône de la Vierge ; on raconte que ce sont les genoux des générations de pénitentes qui lui ont donné cette forme. Dans ce cas, la pratique a engendré une légende exaltant la dévotion de femmes ordinaires. En effet, cette dalle constitue une relique de la dévotion féminine, la mesure de leur sacrifice. Bien que la tâche se transmette d'une génération à l'autre, cette relique renvoie, de manière plus abstraite, à toutes celles qui ont répété les mêmes mouvements pour des raisons similaires; autrement dit, elle évoque l'existence d'une communauté homogène et légendaire des femmes, dont les actions ont convergé pour produire ce résultat.

Même si chaque pèlerine effectue ses génuflexions selon son propre rythme et après un voeu personnel, elles en arrivent finalement à dépasser leur individualité et atteindre un état de fusion, non seulement entre elles, mais aussi avec la Vierge. Parallèlement, leurs actes dévotionnels, même quand ils sont standardisés et répétés de façon automatique, laissent toujours un espace ouvert à l'expression individuelle. Ainsi, j'ai vu une femme saluer l'icône en faisant le signe de croix sur le cou de la Vierge, puis en le répétant sur son propre front. Étant donné que le cou et le visage de la Vierge constituent les seules parties de son "corps" peint qui ne sont pas couvertes par le repoussé métallique, nous pouvons penser que le geste inhabituel de la femme avait pour but de mettre en contact "physique» la chair de la Vierge et sa propre chair. C'est ainsi que les dévotes parviennent à instaurer des liens de complicité avec l'icône : plus on improvise, plus on personnalise la relation.

Lors de la fête, les femmes alternent expressions individuelles et rituels collectifs. Elles arrivent ainsi à partager le sentiment d'appartenance à un groupe féminin plus large, tout en accomplissant les devoirs religieux nécessaires pour le bien-être de leur propre famille et la transmission de la dévotion à la nouvelle génération.

Si les hommes se fixent pour but de faire de Nissyros le dernier rempart d'une tradition vivante et solidaire, les femmes contribuent à ce que l'île devienne l'endroit que la Vierge choisit pour se manifester chaque 
année. Elles sont impliquées dans le domaine du miraculeux, alors que les hommes se placent du côté de la tradition festive. Par leur rôle de défenseurs des coutumes locales, ceux-ci ne peuvent laisser les femmes monopoliser le champ du religieux; la religion devient alors pour eux un langage à manipuler afin de construire un idéal de masculinité.

La fête religieuse de Nissyros est, sans doute, un des lieux où s'accomplit la construction de la différence entre les sexes : chaque sexe forge ses caractéristiques en s'opposant à l'autre. En même temps, on adopte des comportements qui brouillent les frontières entre les sexes - ce qui entrấne des contradictions apparentes : les niameritisses sont appelées à effacer temporairement leur identité sexuelle, tandis que la population masculine affirme sa virilité en cuisinant pour les autres.

Mis à part la négociation entre sexes et l'accomplissement des voeux personnels ${ }^{13}$, les festivités en l'honneur de sa patronne permettent à la communauté locale de célébrer sa cohésion et sa continuité dans le temps. Des stéréotypes tels que «femmes pieuses-hommes viveurs» font partie de l'image idéalisée que cette communauté choisit de véhiculer. Néanmoins, la réalité contredit une aussi simple répartition des rôles : si les hommes sont, en général, associés à la rationalité et les femmes à l'affectivité, les deux sexes sont ici caractérisés par un comportement émotionnel et excessif. De plus, les hommes font la fête pour exprimer leur dévotion à la Vierge, tandis que les femmes, grâce à leur dévouement religieux, acquièrent un statut public important. En effet, leur foi leur donne un aspect presque héroïque, ainsi que les versets qu'elles chantent à la Vierge le soulignent : "Même si la mer est très agitée, même si le courant rend la traversée aussi difficile que celle d'une montagne, [les niameritisses] se jettent à l'eau afin d'arriver jusqu'à toin.

13 Les hommes font aussi des voeux à la Panayia Spiliani. Ce n'est pas seulement la légende concernant les visiteurs du monastère qui jetaient leurs verres par la fenêtre qui confirme cette tendance. On m'a raconté qu' "un Américain", c'est-à-dire un émigré, dont la Vierge avait exaucé le vœu, a décidé d'offrir chaque année un veau pour sa fête qu'il abat lui-même. Il est clair que ces vœux masculins consolident la figure de l' "homme fêtard". 


\section{Bibliographie}

ALBERT-LLORCA Marlène, 1995, «La Vierge mise à nu par ses chambrières", CLIO, Histoires, Femmes et Sociétés, 2, pp. 201-228.

DUBISCH Jill, 1983, "Greek women : sacred or profane", Journal of modern Greek studies, 1, pp. 185-202.

DUBISCH Jill, 1991, "Gender, kinship, and religion : "Reconstructing" the anthropology of Greece», in Peter Loizos and Evthymios Papataxiarchis (dir.), Contested identities : Gender and kinship in modern Greece, Princeton, Princeton University Press, pp. 29-46.

GEORGIEVA Ivanichka, 2001, «Le feu et la croix : un rite en l'honneur de saint Constantin", Ethnologie française, XXXI, 2, pp. 251-260.

HART Laurie Kain, 1992, Time, religion and social experience in rural Greece, Maryland, Rowman and Littlefield Publishers.

KENTRIS Stavros, 1991, Panayia Spiliani, Nissyros, Éditions du monastère saint de la "Panayia Spiliani».

PAPATAXIARCHIS Evthymios, 1994, «Émotions et stratégies d'autonomie en Grèce égéenne», Terrain, 22, pp. 5-20.

SAKELLARIDIS Kostas, 1982, "Paradosseis kai topikes istories tis Nissyrou" (Traditions et histoires locales de Nissyros), Nissyriaka, vol. VIII, pp. 302-314.

SERAÏDARI, Katerina, 2000, L'éternel présent. Légendaire et culte des icônes en Grèce, EHESS, Toulouse, Thèse non publiée. 